Anales del Seminario de Historia de la Filosofía

ISSN-e 1988-2564

http://dx.doi.org/10.5209/ASHF.63361

\title{
El universal lógico como modus concipiendi en Duns Escoto ${ }^{1}$
}

\author{
Héctor Hernando Salinas Leal ${ }^{2}$
}

Recibido: 06 de junio de 2017 / Aceptado: 19 de febrero de 2018

Resumen: En este trabajo se estudia la concepción escotista del universal entendido como universal lógico y accidente intencional, cuya función cognitiva se entiende como modo de aprehensión o concepción de la esencia o naturaleza. El artículo se concentra en analizar las cuestiones 4 y 5 del comentario de Duns Escoto a la Isagoge. Se subraya la relación de los análisis escotistas con la teoría aviceniana del triple estatuto de la esencia sobre el telón de fondo de la teoría boeciana de los universales. Al final del artículo se presenta brevemente el momento ontológico del universal tal como se puede ver en la teoría de la naturaleza común.

Palabras clave: Juan Duns Escoto; Boecio; Avicena; problema de los universales; filosofía medieval.

\section{[en] Duns Scotus on the logical universal as modus concipiendi}

Abstract: This work studies the scotistic conception of the universal as logical universal and intentional accident, whose cognitive function is understood as the way of apprehension or conception of the essence or nature. The article focuses on analyzing questions 4 and 5 of Scotus commentary on the Isagoge. The article underlines the relationship between Scotus's approach and the Avicennian theory of the triple status of essence taking into account the background of the Boethian theory of universals. The final moment of the article shows briefly the ontological moment of the universal as it can be seen in the theory of the common nature.

Key words: John Duns Scotus; Boethius; Avicenna; problem of universals; medieval philosophy.

Sumario: 1. Introducción; 2. El zócalo porfirio-boeciano; 3. El avicenismo; 4. La ontología del universal lógico; 5. La inteligibilidad del universal; 6. La naturaleza común; 7. Consideraciones finales; 8. Bibliografía.

Cómo citar: Salinas Leal, H.H. (2019): "El universal lógico como modus concipiendi en Duns Escoto", en Revista Anales del Seminario de Historia de la Filosofía 36 (1), 29-48.

1 Este artículo es uno de los resultados del proyecto de investigación 006268: Juan Duns Escoto antes de la univocidad del ser, Inscrito en la Vicerrectoría de Investigación de la Pontificia Universidad Javeriana, Bogotá.

2 Pontificia Universidad Javeriana, Bogotá

hsalinas@javeriana.edu.co

ORCID: https://orcid.org/0000-0002-3381-7442 


\section{Introducción}

¿Qué es un universal? ¿qué es la universalidad? ¿Se trata de un acto del intelecto? ¿De la producción de un concepto sin fundamento in re? ¿Se trata acaso de un rasgo común a las cosas que se transfiere en el acto de conocer a los conceptos y las palabras? Al menos en el Occidente Latino, la pregunta por la naturaleza de lo universal está asociada a un librito introductorio concebido sin mayores pretensiones, una introducción, Isagoge, a las Categorías de Aristóteles, escrita por Porfirio, el neoplatónico, el discípulo y editor de las obras de Plotino ${ }^{3}$.

Esta tradición inaugurada por Porfirio sufrirá múltiples variaciones a lo largo de los siglos. En efecto, la Isagoge tuvo un destino inesperado, al servir de zócalo a la discusión sobre la naturaleza de los universales en el Occidente Medieval ${ }^{4}$. Muy a pesar de su intención explícita de no ahondar en la solución de las dificilísimas cuestiones que plantea sobre el estatuto de los géneros y las especies, gracias a la traducción latina ${ }^{5} \mathrm{y}$ a los dos comentarios ${ }^{6}$ de Boecio, el problema de los universales obtiene su fisonomía medieval latina a partir de esta primera recepción boeciana de la Isagoge.

Aquí pretendo explicitar una de esas mutaciones del motivo porfiriano atendiendo a la concepción escotista del universal contenida precisamente en su comentario a la Isagoge de Porfirio. En este trabajo estudiaremos fundamentalmente las cuestiones 4 y 5 del comentario ${ }^{7}$, en las que Escoto se pregunta qué es un universal y cómo se conoce. Mostraremos la dependencia que guarda su enfoque con respecto a la teoría aviceniana del triple estatuto de la esencia. Veremos cómo, con respecto a la esencia, el universal es su modo de aprehensión (modus concipiendi) por parte del intelecto.

Sin embargo, en lo que sigue inmediatamente, comenzaremos regresando al punto de partida de esta tradición medieval, precisamente en el dispositivo porfirioboeciano en el que se plantea el problema medieval de los universales.

\section{El zócalo porfirio-boeciano}

En la versión castellana de García Norro y Rovira, así reza el cuestionario de Porfirio:

3 En castellano contamos con dos ediciones recientes: Porfirio, Isagoge, Introducción, traducción y notas de J. J. García Norro y R. Rovira, Anthropos, Barcelona 2003 y Aristóteles, Categorías/De interpretatione y Porfirio, Isagoge, introducción traducción y notas de L. M. Valdés Villanueva, Tecnos, Madrid 2012.

4 Cf. Libera, A. de "Introduction", en Porphyre, Isagoge, texto griego y latino, traducción francesa de A. de Libera y A.-Ph. Segonds, introducción y notas de A. de Libera, Vrin, París 1998, pp. LXII-CXL y, del mismo autor, La Querelle des Universaux. De Platon à la fin du Moyen Age, Seuil, París 1996, pp. 128-130.

5 Porphyrius, Liber praedicabilium (Isagoge) Translatio Boethii, en Aristoteles Latinus, Categoriarum supplementa. Porphyrii Isagoge, Translatio Boethii, et Anonymi Fragmentum vulgo vocatum «Liber sex principiorum», Minio-Paluello L. y Dod, B. G. (eds.), «Aristoteles Latinus, I 6-7», Desclée De Brouwer, BrujasParís 1966, pp. 1-31.

6 Boecio, In Isagogen Commenta, S. Brandt (ed.), «Corpus Scriptorum Ecclesiasticorum Latinorum, 48», F. Tempsky y G. Freytag, Viena-Leipzig 1906.

$7 \quad$ Ioannis Duns Scoti, Quaestiones In Librum Porphyrii Isagoge, en R. Andrews et al. (eds.), Opera philosophica tomo I, The Franciscan Institut, St. Bonaventure (NY) 1999, pp. 21-28. En adelante se cita así: Duns Scotus, In Porph., q. 1 n. 1 (OPh, I, 3), comentario a la Isagoge, cuestión 1, parágrafo 1, en las Opera Philosophica de Escoto, volumen I, página 3. En este artículo, salvo mención contraria, todas las traducciones del latín son del autor. 
Así, pues, sobre los géneros y las especies declinaré hablar tanto de si subsisten o son puros y simples pensamientos, como de si son subsistentes corpóreos o incorpóreos, como también de si están separados o si son subsistentes en las cosas sensibles y dependen de ellas, por ser el tratamiento de todo esto profundísimo y requerir un examen mayor ${ }^{8}$.

Una de las virtudes del cuestionario de Porfirio radica precisamente en su estatuto de puro cuestionario. No hay una solución a estas tres preguntas en la Isagoge y sin embargo hay una invitación implícita a responderlas. De allí que la Isagoge funde un espacio de posibilidades filosóficas para sus futuros lectores. Es un gesto que Boecio reconoce plenamente: «Porfirio agregó aquello mismo cuyo cuestionamiento prometió diferir. Y esto, para no abrumar al lector al tratar estos asuntos de modo oscuro y profundo; y sin embargo, que robustecido por la ciencia, el lector pueda reconocer aquello que podría justamente investigar» ${ }^{9}$.

Con todo, este espacio no está del todo abierto. Menciono al menos dos supuestos de este cuestionario. El primero, que géneros y especies son efectivamente «puros y simples pensamientos» (in solis nudis purisque intellectibus). Porfirio no pone en duda, para decirlo en términos más contemporáneos, el carácter mental de géneros y especies. Busca, sobre este supuesto, establecer si ellos se dan también de algún modo fuera de la mente, es decir, si se fundan en algo fuera de la mente (bajo algún tipo de subsistencia y relación con las cosas sensibles). En segundo lugar, Porfirio guía al lector a partir de una distribución disyuntiva de las soluciones posibles. Por ejemplo, que además de ser pensamientos, los géneros y especies subsistan de acuerdo a dos modos posibles de subsistencia mutuamente excluyentes (ser corpóreos o incorpóreos); y que de ser incorpóreos, se relacionen con las cosas sensibles también de dos modos mutuamente excluyentes (separados de o subsistentes en ellas). En otros términos, el problema se formula y se resuelve durante muchos siglos, a partir de este cuestionario en su interpretación boeciana, en términos de una relación diádica entre el universal en la mente y su fundamento en la realidad extramental.

En efecto, en su segundo comentario a la Isagoge, Boecio también heredará a la filosofía medieval la primera solución al cuestionario porfiriano, sobre su propia interpretación de las condiciones del problema, pero preservando de otro modo, como veremos, el gesto inaugural de la Isagoge, al no comprometer una posición personal sobre el asunto ${ }^{10}$. Sobre su interpretación del problema señalo solamente dos elementos. Boecio interpreta la primera pregunta como una alternativa entre la intelección de aquello que se encuentra constituido en la naturaleza de las cosas (en caso de que géneros y especies sean algo más que pensamientos) o aquello que resulta de la actividad de la «vacía imaginación» y no es (o sólo es en tanto producto de ella $)^{11}$. En segundo lugar, con respecto a la tercera pregunta, Boecio introduce

8 Porfirio, Isagoge, op. cit., pp. 2-3. El texto de la traducción de Boecio, Liber praedicabilium (Isagoge) Translatio Boethii (AL, I 6-7, p. 5), dice así: «Mox de generibus et speciebus illud quidem sive subsistunt sive in solis nudis purisque intellectibus posita sunt sive subsistentia corporalia sunt an incorporalia, et utrum separata an in sensibilibus et circa ea constantia, dicere recusabo (altissimum enim est huiusmodi negotium et maioiris egens inquisitionis)».

9 Boecio, In Isagogen Porphyrii Commenta. Ed. Secunda, (ed. Brandt, I, 10, 159-160): «Id ipsum cuius exequi quaestionem se diferre promisit, addidit. Ut de his minime obscure penitusque tractando nec lectori quicquam obscuritas offunderet et tamen scientia roboratus quid quaeri iure posset agnosceret».

10 Cf. Marenbon, J. Boethius, Oxford University Press, Oxford 2003, pp. 26 ss.

11 Boecio, In Isagogen Porphyrii Commenta. Ed. Secunda, (ed. Brandt, I, 10, 160): «Quarum prima est huius modi. Omne quod intellegit animus aut id quod est in rerum natura constitutum, intellectu concipit et sibimet 
una distinción que determinará su propia solución. Hay dos clases de entidades incorpóreas: (a) las que pueden existir separadas de los cuerpos y perdurar en su incorporalidad y (b) las que siendo incorpóreas no pueden subsistir separadas de los cuerpos. Para el primer caso, Boecio menciona a Dios, la mente y el alma; y para el segundo, la línea, la superficie, el número y las cualidades singulares ${ }^{12}$. Este segundo grupo de entidades se consideran incorpóreas por carecer de tridimensionalidad. Sin embargo, ni pueden separase de los cuerpos en que se hallan, ni permanecen o subsisten si se separan de ellos.

Sobre estas condiciones se plantea la pregunta que aglutina todo el problema en torno a géneros y especies: o existen y subsisten, o son formados únicamente por el intelecto. $\mathrm{O}$, en los términos antes utilizados por Boecio, lo inteligido se funda en un elemento constitutivo de la naturaleza de las cosas o no es más que una pura ficción de la imaginación.

Boecio presenta algunos argumentos contra la existencia de los géneros y especies así entendidos. Recojo los dos más relevantes. El primero descansa en la tensión entre unidad y multiplicidad. Si el universal, como un todo, es según su definición común a muchos simultáneamente, de allí se sigue que no pueda tener unidad (pues se disgrega en la multiplicidad a la que es común) y, en último término, tampoco realidad, si la realidad de una entidad depende de su unidad ${ }^{13}$. En ese caso ni géneros ni especies existen, pues su grado de comunidad supone una multiplicidad que compromete su unidad.

Boecio también presenta la objeción conversa. Si géneros y especies tienen unidad, no pueden ser comunes a muchas realidades. Boecio define los tipos de comunidad para descartar sistemáticamente géneros y especies comprendidos como unidad. Así se excluye que algo sea común a muchos por división de sus partes, pues el todo no sería común. Se excluye también la comunidad por sucesión temporal, pues el todo no sería común al mismo tiempo. Y, se excluye la comunidad plena, cuando esta no involucra la esencia de las cosas en las que se da dicha comunidad, pues no sería una comunidad de género o especie ${ }^{14}$.

De esta doble argumentación se sigue la imposibilidad de que géneros y especies existan fuera de la mente, pues su comunidad como universales se entiende precisamente como completa (se da plenamente en cada uno de los singulares de que se predica), simultánea (se da en todos ellos al mismo tiempo) y sustancial (constituye un elemento esencial a ellos) ${ }^{15}$.

Sin embargo, Boecio también presenta un argumento a favor de la existencia de los universales. Suponiendo que géneros y especies sean aprehendidos por conceptos (intellectibus), deben depender de algún «sujeto» del que se toman. Y ellos se conciben sea como se hallan en dicho sujeto o de otro modo distinto a como se

ratione describit aut id quod non est, uacua sibi imaginatione depingit».

12 Boecio, In Isagogen Porphyrii Commenta. Ed. Secunda, (ed. Brandt, I, 10, 160-161): «Duae quippe incorporeum formae sunt, ut alia praeter corpora esse possint et separata a corporibus in sua incorporalitate perdurent, ut deus, mens, anima, alia uero cum sint incorporea, tamen praeter corpora esse non possint, ut linea uel superficies uel numerus uel singulae qualitates».

13 Boecio, In Isagogen Porphyrii Commenta. Ed. Secunda, (ed. Brandt, I, 10, 161): «Omne enim quod commune est uno tempore pluribus, id unum esse non poterit».

14 Boecio, In Isagogen Porphyrii Commenta. Ed. Secunda, (ed. Brandt, I, 10, 162).

15 Boecio, In Isagogen Porphyrii Commenta. Ed. Secunda, (ed. Brandt, I, 10, 163): «Nam ita commune esse debet, ut et totum sit in singulis et uno tempore et eorum quorum commune est, constituere ualeat et formare substantiam». 
hallan en él. Como es evidente, la premisa fundamental aquí es que no hay concepto que no provenga de un sujeto. Así pues, si el concepto corresponde al modo como se da en el sujeto, también tendría realidad fuera de la mente. Por el contrario, si no corresponde, no la tendría y el concepto sería vacío, pues aunque tomado de un sujeto, el concepto no correspondería a su modo de darse en aquel. Lo que conduce de nuevo a la pura intelectualidad o ficcionalidad de géneros y especies ${ }^{16}$.

Porfirio no hizo ninguna de estas consideraciones en su planteamiento de las preguntas en la Isagoge. Boecio, en cambio, construye la problemática y sobre ella ofrece una respuesta, nos dice, en acuerdo con Alejandro de Afrodisia ${ }^{17}$. La distinción principal en el camino de su solución consiste en mostrar que aunque el intelecto conciba algo de modo distinto a como se da en la realidad fuera de la mente, de allí no se sigue que dicho concepto sea falso o erróneo. Distingue pues Boecio entre (a) aquello que concibe el intelecto por abstracción y separación de lo que está unido a lo sensible (concepto que puede ser verdadero, como en el caso de la línea pensada sin el cuerpo en el que se da) y (b) el concepto del intelecto producido por composición de realidades que no se dan juntas fuera de la mente (como la del caballo y el hombre que configuran al centauro). El primer caso le permite mostrar que se pueden distinguir el modo de subsistencia y el modo de intelección de una realidad, pues, como en el caso de la línea, ella subsiste en un cuerpo singular, pero se la concibe separada de él y universalmente ${ }^{18}$. Así pues, el recurso a la abstracción permite mostrar que hay conceptos que aunque no corresponden plenamente a la realidad extramental, no por ello pueden calificarse de falsos o ficticios, pues la abstracción es precisamente el modo a través del cual el intelecto conoce lo que subsiste en la realidad como género o especie, despojándolo de sus condiciones materiales y por lo tanto universalizándolo ${ }^{19}$.

Con esta distinción, Boecio da respuesta a las tres preguntas del cuestionario Porfiriano: «Los géneros y las especies subsisten de un modo, pero son inteligidos de otro modo. Son incorpóreos, pero subsisten unidos a los sensibles, en los sensibles. Sin embargo, son inteligidos, como si subsistieran en sí mismos y no como si tuvieran su ser en otros $»^{20}$. Según esta solución, los géneros y las especies son algo más que pensamientos. Son, según la distinción introducida por Boecio, realidades incorpóreas que subsisten en las cosas sensibles y no pueden subsistir separadas de ellas, pero que son inteligidas separadas de ellas, por abstracción, como si tuvieran una subsistencia independiente. Pero este último punto es un efecto adicional al acto de abstracción por el que el intelecto concibe el universal y no corresponde a la naturaleza del universal en tanto unido a las cosas sensibles. Por ello, la solución debe comportar los dos elementos, tanto el modo en que subsisten como el modo en que son inteligidos los universales.

16 Boecio, In Isagogen Porphyrii Commenta. Ed. Secunda, (ed. Brandt, I, 10, 163-164).

17 Libera, A. de L'art des géneralités. Théories de l'abstraction, Aubier, París 1999, pp. 159-175, propone un exhaustivo análisis y una nueva interpretación del rol de Boecio en la transmisión del pensamiento de Alejandro de Afrodisia con respecto al problema de los universales. De hecho, en su interpretación, el tratamiento boeciano del problema de los universales es alejandriniano.

18 Boecio, In Isagogen Porphyrii Commenta. Ed. Secunda, (ed. Brandt, I, 11, 164-165).

19 Cf. Marenbon, J. Boethius, op. cit., p. 27.

20 Boecio, In Isagogen Porphyrii Commenta. Ed. Secunda, (ed. Brandt, I, 11, 167): «Ipsa enim genera et species subsistunt quidem alio modo, intelleguntur uero alio, et sunt incorporalia, sed sensibilibus iuncta subsistunt in sensibilibus. Intelleguntur uero ut per semet ipsa subsistentia ac non in aliis esse suum habentia». 
Boecio cierra su solución con dos observaciones que cumplen la función de situar su interpretación y de, paradójicamente, reabrir la discusión. En primer lugar, Boecio presenta en unas cuantas líneas las posiciones de Platón y Aristóteles. Para Platón, dice, los géneros y las especies no sólo se inteligen como universales, sino que también son y subsisten separados de los cuerpos. Aristóteles, por su parte, considera que los géneros y especies son inteligidos en tanto incorpóreos y universales; pero subsisten en las cosas sensibles ${ }^{21}$. En segundo lugar, y aquí Boecio, a su manera, repite el gesto porfiriano de suspender la posición de una solución definitiva de su parte, afirma que no tuvo la intención de escoger entre la solución de Platón y la de Aristóteles, «pues esto corresponde a una filosofía más alta» (altioris enim est philosophiae ${ }^{22}$. Sin embargo, Boecio afirma seguir la posición de Aristóteles, no porque la apruebe, sino en virtud de la intención con la que Porfirio escribió la Isagoge. Es decir, en tanto la Isagoge se presenta como una introducción a las Categorías cuyo autor es Aristóteles ${ }^{23}$. En otros términos, Boecio parece considerar que su labor como comentarista de la Isagoge lo limita a seguir la lógica del texto comentado incluso en aquello que el texto no dice, como es aquí el caso respecto de las preguntas que Porfirio formula pero se abstiene de responder.

Sobre estos dos elementos, el cuestionario de Porfirio y la solución de Boecio, se construye pues la tradición medieval latina del problema de los universales. Solo hasta el siglo XII se introducirán nuevas variables a la problemática porfirio-boeciana. Y una de las más importantes es precisamente el tratamiento aviceniano del problema. Quizá valga la pena recordar aquí a Alain de Libera, quien sintetiza finamente esta modificación del escenario medieval, no solo con respecto a la pregunta ¿qué es un universal?, sino también con respecto al conjunto de las problemáticas filosóficas tal como fueron tratadas antes (en el período boeciano) y después del siglo XII: «Hasta la segunda mitad del siglo XII, es decir, hasta la traducción de nuevas fuentes árabes (Avicena) y aristotélicas (Logica nova, De anima, Metaphysica vetus), es con Boecio, y únicamente con Boecio, que los medievales tuvieron acceso a los datos filosóficos de la Antigüedad y de la Antigüedad Tardía» ${ }^{24}$.

\section{El avicenismo}

Así pues, cuando siete siglos más tarde, en la década de 1290, Escoto redacta su comentario a la Isagoge, su interpretación del problema depende de una tradición que se ha enriquecido con un conjunto de tesis de origen aviceniano. En efecto, la recepción latina de Avicena supuso en este punto una reconfiguración del problema y una concentración de la pregunta por el estatuto del universal en el ámbito de la actividad del intelecto. $\mathrm{O}$, dicho en otros términos, esta tradición permitió a Escoto

21 Boecio, In Isagogen Porphyrii Commenta. Ed. Secunda, (ed. Brandt, I, 11, 167): «Sed Plato genera et species ceteraque non modo intellegi uniuersalia, uerum etiam esse atque praeter corpora subsistere putat, Aristoteles uero intellegi quidem incorporalia atque uniuersalia, sed subsistere in sensibilibus putat».

22 Boecio, In Isagogen Porphyrii Commenta. Ed. Secunda, (ed. Brandt, I, 11, 167): «Quorum diiudicare sententias aptum esse non duxi, altioris enim est philosophiae».

23 Boecio, In Isagogen Porphyrii Commenta. Ed. Secunda, (ed. Brandt, I, 11, 167): «Idcirco uero studiosius Aristotelis sententiam executi sumus, non quod eam maxime probaremus, sed quod hic liber ad Praedicamenta conscriptus est, quorum Aristoteles est auctor».

24 Libera, A. de La Querelle des Universaux, op. cit., p. 128. 
distinguir, de un lado, el universal como modo de aprehensión de la esencia en el intelecto (modus concipiendi) y, del otro, la esencia en su indiferencia, gracias a la teoría aviceniana sobre el triple estatuto de la esencia.

Por ejemplo, en su Logyca, una cortísima sección del comentario a la Isagoge traducido al latín ${ }^{25}$, Avicena ofrece una de sus formulaciones sobre el triple estatuto de la esencia. Según este triple estatuto, la esencia puede considerarse (a) en sí misma, (b) en tanto se encuentra individuada en los singulares y (c) en tanto es concebida universalmente por el intelecto. Con esta distinción, Avicena separa la dimensión de lo universal, puramente lógica y producto del intelecto, de la dimensión de la esencia en tanto tal, indiferente a su darse en el individuo o en el intelecto. Con ello, la universalidad se distingue de la esencia, de lo universalizado por el intelecto. Si la esencia es la misma en los tres estados, la diferencia es introducida precisamente por su distinción tanto con respecto a la individuación como a la universalización. He aquí el pasaje en cuestión, en el que Avicena distingue al animal en tanto tal, de su individuación en lo sensible y de su universalización en el intelecto:

Animal es por sí algo, y es el mismo si es sensible o si es un concepto en el alma. En sí, sin embargo, ni es universal, ni es singular. En efecto, si fuera universal, de tal modo que la animalidad en tanto animalidad fuera universal, se seguiría necesariamente, por el contrario, que ningún animal sería singular, y todo animal sería universal. Pero si animal en tanto animal fuera singular, sería imposible que hubiera más de un singular, a saber, el mismo singular al cual pertenece la animalidad y sería imposible que otro singular fuera animal. $^{26}$

Subrayemos que animal no solo es distinto a su darse sensible o inteligible, sino que es anterior a ambos. De aquí se sigue una doble transformación. La metafísica será metafísica de las esencias en tanto tales y la lógica será ciencia de las objetos constituidos por las operaciones propias del intelecto. Por ello, la lógica se concebirá como scientia intencionalis ${ }^{27}$. Escoto, heredero de Avicena, forma parte de este movimiento de esencialización de la metafísica ${ }^{28} \mathrm{y}$ de intencionalización de la lógica.

En ese horizonte, el comentario de Escoto a la Isagoge contiene dos momentos introductorios bien diferenciados. En primer lugar, las cuestiones 1 y 2 ofrecen una

25 Cf. Janssens, J. "Latin translations of Ibn Sīnā (Avicenna)", en Lagerlund, H. (ed.), Encyclopaedia of Medieval Philosophy. Philosophy between 500 and 1500, Springer, Dortrecht 2011, pp. 522-527.

26 Avicenna, Logyca, pars III (ed. Venise, 1508, 12ra): «Ponamus autem in hoc exemplum generis dicentes: quod animal est in se quodam: et idem est utrum sit sensibile: aut sit intellectum in anima. In se autem huius: nec est universale: nec est singulare. Si enim in se esset universale: ita quod animalitas ex hoc quod est animalitas est universalis: oporteret nullum animal esse singulare: sed omne animal esset universale. Si autem animal ex hoc quod est animal esset singulare: impossibile esset esse plus quam unum singulare, scilicet, ipsum singulare cui ( ?) animalitas; et esset impossibile alius singulare esset animal». Cf. también Avicenna, Philosophia prima sive scientia divina, V, 1 (AvL, II, 228 ss.), para el pasaje con la esencia más famosa de la metafísica aviceniana: equinitas est equinitas tantum. Encontraremos más adelante una referencia directa de Escoto a este pasaje, en su tratamiento de la naturaleza común.

27 Cf. Salinas Leal, H. H., “Observaciones sobre la constitución medieval de la lógica: de scientia sermocinalis a scientia rationalis", en Universitas Philosophica, 64 (32), enero-junio, 2015, pp. 96-111; Kretzmann, N., "History of Semantics", en Borchert, M. (ed.), Encyclopedia of Philosophy, 2a ed., vol. 8, Macmillan, New York 1967/2005, p. 766.

28 Cf. Sobre este punto, sigue siendo sumamente instructivo el capítulo 3 "Esencia y existencia” de Gilson, É. El ser y los filósofos, Ediciones Universidad de Navarra, Pamplona 2001, pp. 107-146. 
demostración de la cientificidad de la lógica ${ }^{29}$ y la cuestión 3 presenta la determinación de su objeto propio: el silogismo ${ }^{30}$. En segundo lugar, un brevísimo preámbulo a las cuestiones consagradas al estudio de los universales, justo al comienzo de la cuestión 4, inaugura a su vez el cuestionamiento sobre la cientificidad del tratamiento porfiriano de los universales. En otros términos, si el primer momento tiene por fin demostrar la cientificidad de la lógica y su objeto, el segundo se ocupa de demostrar la cientificidad del estudio de los universales y su objeto en la Isagoge.

Propuesto como umbral a la investigación sobre los universales, este preámbulo introduce las distinciones iniciales que permitirán a Escoto exponer varias tesis centrales de su filosofía de la lógica. En primer lugar, (a) Escoto elabora una determinación de la ontología del universal lógico en tanto ente inteligible y accidente intencional en las cuestiones 4 y 5 ; en segundo lugar, (b) ofrece una demostración y una explicación de la naturaleza de la predicación lógica en la cuestión 6 ; y finalmente, (c) determina, en las cuestiones 7 y 8 , el universal como objeto propio de la ciencia de los predicables expuesta en la Isagoge de Porfirio, precisamente al acentuar el carácter unívoco del concepto de «universal» frente a la diversidad de los cinco predicables. En este trabajo, como ya lo anunciamos, nos ocuparemos únicamente de explicitar el primero de estos tres puntos ${ }^{31}$.

\section{La ontología del universal lógico ${ }^{32}$}

Comencemos por leer el preámbulo a la cuestión 4 (Utrum universale sit ens) y su distinción de los tres sentidos del término «universal» ${ }^{33}$ :

Hay que investigar específicamente sobre el universal. Y puesto que lo que se dice por un nombre precede a toda cuestión, hay que notar que <el término> «universal», así como el resto de $<$ términos $>$ concretos, se toma en tres acepciones. A veces $<\mathrm{a}>$ se toma por un sujeto (pro subiecto), es decir, por una cosa de primera intención (pro re primae intentionis), a la que se aplica la intención del universal, y de esta manera el universal es el primer objeto del intelecto. A veces $<\mathrm{b}>$ se toma por una forma, es decir, por una cosa de segunda intención (pro re secundae intentionis) causada por el intelecto y aplicable a las cosas de primera intención, y así el lógico trata propiamente del universal. En tercer lugar $<\mathrm{c}>$ se toma por el agregado de un sujeto y una forma (pro aggregato ex subiecto et forma), y este es un ente por accidente, porque aglutina (aggregat) naturalezas diversas

29 Duns Scotus, In Porph., qq. 1-2 (OPh, I, 3-10). Cf. Salinas Leal, H. H., "La determinación de la lógica como ciencia común intencional en Duns Escoto", en Franciscanum, LVIII (165), enero-junio 2016, 51-86, donde ofrezco un análisis y una traducción castellana de estas dos cuestiones. Sobre la teoría de la ciencia en Duns Escoto son de gran provecho Demange, D., Jean Duns Scot. La théorie du savoir, Vrin, París 2007, pp. 77-114 y Newton, L1. A. "Duns Scotus's Account of a Propter Quid Science of the Categories", en Newton, L1. A. (ed)., Medieval Commentaries on Aristotle's Categories, Brill, Leiden-Boston 2008, pp. 221-258.

30 Duns Scotus, In Porph., q. 3: «Utrum syllogismus sit subiectum logicae» (OPh, I, 11-20).

31 Duns Scotus, In Porph., qq. 4-5 (OPh, I, 21-28).

32 Los numerales 4 y 5 de este artículo recogen parcialmente temas desarrollados en las secciones II, 1 y II, 2 de mi tesis doctoral inédita Duns Scot avant l'univocité de l'étant: études logiques, sémantiques et métaphysiques defendida el 13 de noviembre de 2014 en la École Pratique des Hautes Études (EPHE) de París.

33 Cf. sobre este preámbulo los análisis de Noone, T. B. "Universals and Individuation”, en Williams, T. (ed.), Cambridge Companion to Duns Scotus, Cambridge University Press, Cambridge 2003, p. 106 y Demange, D., op. cit., p. 265. 
a partir de las cuales no se produce una única < realidad> por sí (unum per se); y de este modo, este no hace parte de la consideración de ningún experto (artificis), pues no hay ciencia de un ente por accidente, según Aristóteles en el libro VI de la Metafísica (E, 2, 1027a20-26), porque un ente por accidente no es definible. En consecuencia, en lo que sigue solo se hará un discurso sobre el universal de acuerdo a la segunda acepción ${ }^{34}$.

En principio, dejando a un lado la tercera acepción en tanto carece de interés científico para Escoto, observamos una relación recíproca entre las dos primeras acepciones construida alrededor de los conceptos de «cosa de intención primera»y «cosa de intención segunda». Escoto (a) toma al sujeto o cosa de intención primera como aquello a lo que se aplica la intención universal y (b) toma la forma o cosa de intención segunda como aquella que es aplicable a la cosa de intención primera o sujeto.

Recordemos que Escoto distingue entre primeras y segundas intenciones, apelando también a un vocabulario heredado de Avicena ${ }^{35}$ y con un inmenso porvenir en la filosofía medieval y moderna ${ }^{36}$. Las primeras intenciones son conceptos de cosas (o esencias), mientras las segundas intenciones son conceptos de conceptos. Por ejemplo, el concepto de "hombre" remite a la naturaleza del hombre vista en su indiferencia con respecto a su captación universal por el intelecto ${ }^{37} \mathrm{o}$ a su individuación en el sujeto concreto ${ }^{38}$. Por su parte, los conceptos de "género" o de "diferencia" remiten a un modo bajo el cual el intelecto aprehende los conceptos de primera intención. Una de estas segundas intenciones es el concepto «universal».

Dicho esto, según el preámbulo que comentamos, esta «cosa aplicable» es una intención segunda o intención universal. La relación se da pues entre la universalidad aplicable y la naturaleza que recibe esta universalidad. Dos observaciones adicionales se imponen. La «cosa de intención primera», la esencia en su indeterminación, recibe la universalidad y, así considerada, se constituye como objeto primero del intelecto; por su parte, «la cosa de intención segunda», causada por el intelecto, y portadora de

34 Duns Scotus, In Porph., q. 4 Prooemium (OPh, I, 21): «In speciali quaerendum est de universali. Et quia omnem quaestionem praecedit quid dicitur per nomen, ideo notandum est quod 'universale', sicut et cetera concreta, sumitur tripliciter. $<\mathrm{a}>$ Quandoque pro subiecto, scilicet pro re primae intentionis cui applicatur intentio universalis, et hoc modo universale est primum obiectum intellectus. $<b>$ Quandoque sumitur pro forma, scilicet pro re secundae intentionis, causata ab intellectu et applicabili rebus primae intentionis, et sic loquitur logicus proprie de universali. $<\mathrm{c}>$ Tertio modo pro aggregato ex subiecto et forma, et illud est ens per accidens, quia aggregat diversas naturas ex quibus non fit unum per se; et sic non est de consideratione alicuius artificis, quia de ente per accidens, per Aristotelem VI Metaphysicae, nulla est scientia, quia non est definibile. Tantum igitur de cetero fiet sermo de universali secundo modo sumpto».

35 Avicenna, Philosophia prima sive scientia divina, I, 2 (AvL, I, 10): «Subiectum vero logicae, sicut scisti, sunt intentiones intellectae secundo, quae apponuntur intentionibus intellectis primo, secundum hoc quod per eas pervenitur de cognito ad incognitum»: «En efecto, el objeto de la lógica, como sabes, son las intenciones inteligidas en segundo lugar, ellas se aplican a las intenciones inteligidas en primer lugar, en tanto que por ellas se llega de lo conocido a lo desconocido».

36 Cf. Rijk, L. M. de, "A Study on the Medieval Intentionality Debate up to ca. 1350", en Giraldus Odonis O. F. M., Opera Philosophica Vol. II: De Intentionibus, de Rijk, L. M. (ed.), Brill, Leiden-Boston 2005, pp. 17-376.

37 Por ello, la universalidad es exterior a la naturaleza, pues no está incluida en su definición. Como lo veremos, la universalidad se entiende aquí como el modo bajo el cual el intelecto aprehende una naturaleza (modus concipiendi) y no como la naturaleza misma en tanto universal.

38 Aclararemos más adelante que esta anterioridad e indiferencia no deben entenderse en el sentido de que la naturaleza pueda darse independientemente de sus instancias concretas, se trata de una anterioridad y una indiferencia definicionales, pues la naturaleza tampoco incluye la instanciación en los particulares como parte de su definición. 
la universalidad y de su aplicabilidad, constituye la acepción del término «universal» propia del discurso científico de la lógica.

El preámbulo distingue pues tres regiones en las que se trata del «universal»: (a) el orden de las esencias y de las «cosas de primera intención» que constituyen el objeto primero del intelecto, (b) el orden de las segundas intenciones, causadas por el intelecto y portadoras de la universalidad. Este orden, además, define el dominio general de consideración de la ciencia lógica. Y, por último, (c) el espacio indefinible y, por lo tanto, fuera del alcance del conocimiento científico, del singular en su concreción y su composición. De aquí que el preámbulo asocie la noción de «universal» a la de esencia en el sentido de su triple estatuto.

Sin embargo, hacia el final del preámbulo, Escoto nos dice que el comentario únicamente elaborará un discurso (sermo) sobre el «universal» tomado en el segundo sentido expuesto, el de la forma o segunda intención «causada por el intelecto y aplicable a las cosas de intención primera». En otros términos, el universal será tratado exclusivamente desde el punto de vista lógico.

Vale subrayar que ya en la cuestión 3 de este comentario, en la que Escoto se pregunta por el objeto de la ciencia lógica (subiectum logicae), se consideraron las segundas intenciones como un candidato posible para cumplir ese rol ${ }^{39}$. Sin embargo, aunque pertenecen sin equívoco al espacio lógico, las segundas intenciones no pueden ser el objeto de la lógica, pues para esta ciencia, ellas son «más comunes que su objeto (communius subiecto), así como nosotros afirmamos que toda ciencia se ocupa del ente porque ninguna se ocupa del no-ente ${ }^{40}$. En efecto, Escoto ve en las segundas intenciones una suerte de nivel conceptual transversal y generalísimo al dominio lógico, pues todo concepto lógico, incluido el del silogismo, que corresponde al del objeto propio de la lógica, es una segunda intención, de modo que esta es más general que aquél, en el orden de la generalidad lógica. En ese sentido, vemos en las intenciones segundas el objeto común de la lógica, así como Escoto ve en el silogismo su objeto propio.

Dicho esto, en el espacio del análisis propio al objeto de los universales en la ciencia lógica, las intenciones segundas cumplen la función de distinguir el universal en su sentido lógico de sus dos sentidos ontológicos: (a) el que permite la ciencia de lo real remite a las cosas de primera intención y designa el objeto propio del intelecto aprehendido por una intención universal; y (b) el que remite a los individuos en su concreción e indefinición.

Sin embargo, y este es el aspecto del problema que quisiéramos subrayar en este artículo, Escoto se hace la pregunta a propósito de la ontología del universal tomado en su sentido lógico. En efecto, hay una ontología del universal lógico, pues solo un ente en acto puede mover al intelecto a partir de su pasividad, para actualizarlo en su acto de conocer. Un no-ente, por el contrario, carente de un contenido inteligible o de una noción inteligible, no puede mover al intelecto y por lo tanto no puede asegurar su actualización. Con el universal lógico nos encontramos frente a un ente que posee la densidad ontológica mínima, la de lo inteligible, para ser capaz, para tener la potencia de actualizar al intelecto:

39 Duns Scotus, In Porph., q. 3 n. 8 (OPh, I, 13): «Aliter ponitur quod est de secundis intentionibus applicatis primis, sicut dicit Boethius quia illae sunt communes omnibus determinatis in logica»

40 Duns Scotus, In Porph., q. 3 n. 16 (OPh, I, 15): «Ad auctoritatem Boethii dico quod logica est de illis < secundis intentionibus $>$, sed illa non sunt primum subiectum, sed communius subiecto, sicut dicimus quod omnis scientia est de ente quia nulla de non-ente». Las cursivas son mías. 
Hay que afirmar que el universal es un ente, pues nada se intelige (intelligitur) bajo la razón de no-ente, pues la inteligibilidad mueve al intelecto (intellectus). En efecto, puesto que el intelecto es una potencia pasiva, según Aristóteles en el tercer libro del tratado Sobre el alma (III, 4, 429a 21-24), el intelecto solo obra al ser movido por un objeto; el noente no puede mover algo, pues mover pertenece al ente en acto. En consecuencia, nada es inteligido (intelligitur) bajo la razón de no-ente. Sin embargo, todo lo que es inteligido (intelligitur), es inteligido (intelligitur) bajo la razón de universal. En consecuencia, esta razón $<$ de universal $>$, no es un no-ente ${ }^{41}$.

Pero, ¿qué tipo de ente es el universal lógico? Para responder a esta pregunta, Escoto utiliza (a) la distinción establecida por Aristóteles en el capítulo 2 del tratado sobre las Categorías, entre «ser dicho de un sujeto» y «ser en un sujeto» ${ }^{42}$; y (b) la distinción establecida en el capítulo 5 de la misma obra, entre la substancia primera y la substancia segunda ${ }^{43}$. Con estas distinciones Escoto caracteriza al universal lógico como accidente intencional.

Veamos. La primera distinción recubre dos tipos de accidentes: «ser en un sujeto» es un rasgo de los accidentes reales adheridos a la substancia primera; por su parte, «ser dicho de un sujeto» es un rasgo de los accidentes intencionales predicados de la substancia primera. A partir de esta distinción, el universal lógico se presenta como accidente intencional:

Afirmo que las substancias segundas, tal como son tratadas allí <en el tratado sobre las Categorias $>$, son accidentes no-reales (para $<$ los accidentes reales $>$ se pone el otro miembro, a saber, «ser en») y <accidentes $>$ intencionales, a los que corresponde por sí «ser dicho de». El universal, sin embargo, es lo más común a la substancia segunda, porque la substancia segunda afirma el universal aplicado a algo (aliquid) en el género de la substancia ${ }^{44}$.

Nunca se insistirá demasiado sobre este punto. El universal constituye en efecto el rasgo «más común a la substancia segunda, porque la substancia segunda afirma el universal aplicado a algo (aliquid) en el género de la substancia». En tanto predicada de una substancia primera, la substancia segunda vehicula, al mismo tiempo, la predicación de la universalidad aplicada a esta substancia primera. En otros términos, toda substancia segunda es concebida bajo la razón de universal $\mathrm{y}$, en tanto que tal, esta razón también es aplicada a la substancia primera. Aquí

41 Duns Scotus, In Porph., q. 4 n. 6 (OPh, I, 23): «Dicendum quod universale est ens, quia sub ratione non-entis nihil intelligitur, quia intelligibile movet intellectum. Cum enim intellectus sit virtus passiva, per Aristotelem III De anima, non operatur nisi moveatur ab obiecto; non-ens non potest movere aliquid, quia movere est entis in actu; igitur nihil intelligitur sub ratione non-entis. Quidquid autem intelligitur, intelligitur sub ratione universalis; igitur illa ratio non est non-ens». Interpretamos este pasaje según In Porph. q. 3 n. 14, OPh, I, 14-15, en donde el ente de razón comporta dos dimensiones: (a) al ente considerado por el intelecto (del que hace parte el ente real en tanto inteligible) y (b) al ente causado por el intelecto como sub-conjunto del primero. El ente de razón, sin embargo, no incluye al ente real en su autonomía con respecto al intelecto. Cf. Gilon, O. Indifférence de l'essence et métaphysique chez Jean Duns Scot, Éditions Ousia, Bruselas 2012, p. 366, n. 22.

42 Cf. Aristóteles, Praedicamenta, 2, 1a20-b7 (AL, I², 47-48).

43 Cf. Aristóteles, Praedicamenta, 5, 2a11-19 (AL, I², 48-49).

44 Duns Scotus, In Porph., q. 4 n. 11 (OPh, I, 24): «Dico quod secundae substantiae, ut ibi loquitur, sunt accidentia non realia, de quibus ponit aliud membrum, scilicet 'esse in', sed intentionalia, quibus per se competit 'dici de'. Universale autem est quid communius ad secundam substantiam, quia secunda substantia dicit universale applicatum ad aliquid in genere substantiae». 
observamos dos niveles en el orden del intelecto: uno, propiamente lógico en el que la universalidad es el modo bajo el cual toda substancia es concebida; otro, ontológico, que corresponde a la substancia concebida en tanto distinta de su modo de ser concebida (universalmente).

Es en este contexto que debería comprenderse la definición del universal propuesta en el tratado Sobre la interpretación: «universale quidem quod de pluribus natum est praedicari $\rangle^{45}$, pues el orden intencional proviene de un tipo de accidentalidad fundada sobre el acto de predicar en tanto este se funda en el acto de concebir. La predicación es pues un acto del intelecto y como tal es accidental a las substancias en la medida en que lo que es predicado como universal se agrega a la substancia en el acto mismo de predicar, pues este es externo a la substancia misma y a sus rasgos tanto substanciales como accidentales, en el orden real. Seamos claros en este punto, tanto la predicación esencial en el género de la substancia como la predicación accidental en las otras categorías pertenecen todas al conjunto de los accidentes intencionales desde el momento en que los miramos con los ojos del lógico. Por ello, Escoto concede que aquello que la metafísica trata por sí, la lógica lo trata per accidens, puesto que esta última trata por sí lo que se aplica en tanto que intencional al orden de lo real ${ }^{46} \mathrm{o}$, utilizando la expresión del preámbulo, a las cosas de primera intención.

Esto no quiere decir, y en este punto la lucha permanente de Escoto es el mejor testimonio, que el universal, siendo un ente de razón, y con mayor precisión, una segunda intención, es decir, producido por el intelecto ( $a b$ intellecto), esté confinado o reducido al reino de las ficciones (figmenta). Si así fuera, el universal se convertiría en una pura invención del intelecto sin ninguna correspondencia o vínculo con la naturaleza común ${ }^{47}$.

En efecto, en la cuestión 4, la tercera objeción sostiene que el universal es producido ab intellectu solo y, por lo tanto, que no es más que una ficción ${ }^{48}$. En este pasaje, encontramos por primera vez en el comentario, el aspecto ontológico de la dimensión lógica. El universal, a diferencia del ente de ficción, sostiene una relación con lo real. Escoto habla de una correspondencia entre el universal y algo de la cosa externa. Esta mueve al intelecto a causar tal tipo de intención: el universal ${ }^{49}$.

45 Aristote, De Interpretatione, 7, 17a39-44 (AL, II $\left.{ }^{2}, 45\right)$ : «Llamo universal a lo que es natural que se predique sobre varias cosas» (trad. Candel Sanmartín, p. 44); Auctoritates Aristotelis (ed. J. Hamesse, 305, n. 10): «Universale est quod aptum natum est praedicari de pluribus, et singulare quod non».

46 Este punto es mucho más claro a propósito del doble tratamiento científico de las categorías, cf. In Praed., q. 2 n. 30 (OPh, I, 265): «patet quod quae metaphysicus considerat per se, hic < in logica > considerantur per accidens, quia scilicet per se consideratur aliquid intentionnale applicabile eius»: «es evidente que lo que el metafísico considera por sí, aquí < en lógica> es considerado accidentalmente, a saber porque es considerado por sí algo intencional aplicable a ellas» (trad. Salinas, p. 295). Cf. Boulnois, O. "Les catégories selon Duns Scot", en M. Carbajo Núñez (ed.), Giovanni Duns Scoto: Studi e ricerche nel VII Centenario della sua morte, Vol.1, Roma, Antonianum, Roma 2008, pp. 357-377 y Salinas Leal, H. H., "Naturaleza y unidad lógica de las categorías en Duns Escoto", en Pensamiento: Revista de investigación e información filosófica, 73 (275), enero-abril, 2017, pp. $129-145$.

47 Esta posición hace eco a la solución boeciana y a su consideración del alcance ontológico del universal, al menos en ese punto. Cf. La segunda sección de este trabajo.

48 Duns Scotus, In Porph., q. 4, n. 3 (OPh, I, 22): «Item, aut est a natura, aut ab intellectu. Non a natura, quia tunc esset singulare et terminus transmutationis. Igitur ab intellectu solo; igitur est figmentum et ita non-ens».

49 Duns Scotus, In Porph., q. 4 n. 12 (OPh, I, 25): «Ad tertium dico quod universale est ab intellectu. Cum dicitur 'igitur est figmentum', dico quod non sequitur, quia figmento nihil correspondet extra; universali autem aliquid correspondet extra a quo movetur intellectus ad causandum talem intentionem». 
Aquí encontramos pues uno de los lugares de contacto entre lógica y ontología. Escoto emprende una lucha permanente contra una doble reducción del universal como entidad lógica: (a) reducción a la realidad de tal modo que las entidades lógicas se hacen depender totalmente de las cosas o esencias, dando lugar a la inclusión de la dimensión intencional en la estructura de lo real, limitando de paso la potencia causal del intelecto en tanto que productor de las intenciones lógicas; (b) o reducción a una ficción, en la medida en que las segundas intenciones no tendría ningún vínculo con lo real y solo serían un producto del intelecto. Escoto busca un espacio que ni reduzca el universal lógico a lo real, ni lo libere al capricho de la causalidad del intelecto.

Por ello, el universal es producido por una doble causalidad, el intelecto es su causa eficiente (lo que garantiza su inclusión en el reino de los entia rationis) y la cosa o alguna de sus propiedades es su causa ocasional, material u originaria ${ }^{50}$. Este motivo del análisis escotista de la producción del universal, concentrado en el concepto de occasio, será retomado por la pluma de Escoto, precisamente para evitar los dos extremos ya mencionados.

Sin embargo, la definición ontológica del universal sigue siendo difusa, como una definición negativa en la que el universal no puede ser reducido ni a las cosas de intención primera ni a los figmenta. ¿En qué consiste entonces la naturaleza del universal? Su densidad ontológica parece limitarse, lo que no es poco, justamente a su inteligibilidad. Puesto que el ente y solo el ente es inteligible, el universal pertenece plenamente al orden del ente, precisamente en tanto inteligible.

Esta relación a lo real, en tanto aquello que juega el rol de occasio y, con ello, suscita el movimiento del intelecto en la producción del universal, es un aspecto central de esta producción para sostener, si no la dependencia total, al menos una dependencia parcial del intelecto con respecto a lo real. Bajo ninguna circunstancia el universal puede ser entendido como invención del intelecto. Este es un asunto controvertido, pues algunos intérpretes de Escoto sostienen una forma de autonomía total de la lógica con respecto a lo real ${ }^{51}$. Nosotros no lo vemos de este modo, al menos con respecto a esta fase del pensamiento de Escoto.

\section{La inteligibilidad del universal lógico}

La cuestión 5 desarrolla esta tesis de la inteligibilidad del universal y explica de otro modo la distinción del preámbulo entre los tres sentidos del término «universal». La inteligibilidad le corresponde al universal en lo que es. Así, el universal es inteligible por sí. Es decir, al universal le pertenece una inteligibilidad que no se confunde con la inteligibilidad de la cosa misma que el universal permite aprehender. De hecho, Escoto establece una distinción entre la cosa conocida y el modo bajo el cual ella es conocida. El objeto primero del intelecto, la esencia (quod quid est), se conoce bajo la razón del universal, es decir, el intelecto concibe de modo universal una naturaleza que en sí misma ni es universal, ni particular, sino común. De este modo,

50 Duns Scotus, In Porph., q. 4 n. 12 (OPh, I, 25): «Dico igitur quod effective <intentio> est ab intellectu, sed materialiter sive originaliter sive occasionaliter a proprietate in re; figmentum autem non sic; igitur non est figmentum».

51 Cf. Dahlstrom, D. O. "Signification and Logic: Scotus on Universals from a Logical Point of View", en Vivarium, 18, 1980, pp. 81-111. 
el universal no es esencialmente idéntico a la esencia o naturaleza concebida, sino que, con respecto a ella, el universal corresponde a un modus accidentalis.

Esta distinción es fundamental. De un lado, encontramos la esencia en su desnudez $\mathrm{y}$, del otro, el modo de su aprehensión, el universal. «El intelecto puede conocer la diferencia entre su objeto primero y este modo, porque puede distinguir entre todos aquellas realidades que no son esencialmente idénticas ${ }^{52}$. He aquí un segundo rasgo diferenciador de los dos primeros sentidos del término «universal» presentes en el preámbulo a estas cuestiones. El objeto primero del intelecto, una intención primera, es conocido bajo la razón o el modo de lo universal; por su parte, este modo es una intención segunda y un modo accidental del objeto primero en tanto es aprehendido o concebido por el intelecto. Este objeto primero y su modo, nos dice Escoto, son esencialmente diferentes, y por ello el intelecto puede conocerlos independientemente uno del otro. Pero esto implica, que el intelecto puede conocer en sí mismo el modo de aprehensión. Aquí se encuentra esbozada la justificación epistemológica de la ciencia lógica.

«Por lo tanto, afirma Escoto, el intelecto puede conocer aquel modo o razón universal por sí y bajo su propia razón. De este modo, reflexionando, el intelecto se conoce y conoce su operación y el modo de operar y todas las otras propiedades que se encuentran en él» ${ }^{53}$. La reflexión sobre sí da acceso al conocimiento de las operaciones del intelecto, entre ellas, la operación de constitución del universal y el universal mismo, ahora considerados en sí mismos.

Detengámonos en la tesis operante sobre el objeto propio del intelecto, aquí identificado con la esencia o naturaleza conocida (quod quid est). Esta es la posición inicial de Escoto que contrasta profundamente con la tesis que presenta en su célebre y tardío Quodlibet III $^{54}$. En efecto, en esta obra distingue Escoto tres sentidos de res vel ens de los cuales nos interesa especialmente el primero, en el que "ente" se toma communissime:

Ente, en el primer sentido, se toma, pues, comunísimamente (omnino communissime), y se extiende a todo lo que no incluye contradicción, sea ente de razón (ens rationis) -es decir, algo que sólo tiene ser en el intelecto considerante (habens esse in intellectu considerante)-, sea ente real (ens reale) -es decir, algo que tiene entidad fuera de la consideración del entendimiento (trad. Alluntis, p. 93) $)^{55}$.

52 Duns Scotus, In Porph., q. 5 n. 4 (OPh, I, 27-28): «Universale est per se intelligibile. Quod patet sic: primum obiectum intellectus, scilicet 'quod quid est' intelligitur sub ratione universalis. Illa autem ratio non est idem essentialiter cum illo 'quod quid est', sed modus eius accidentalis. Igitur intellectus potest cognoscere differentiam inter suum primum obiectum et illum modum, quia potest distinguere inter omnia quae non sunt essentialiter eadem». Las cursivas son mías.

53 Duns Scotus, In Porph., q. 5 n. 4 (OPh, I, 28): «Igitur intellectus potest cognoscere illum modum sive rationem universalis per se et sub propria ratione. Hoc modo, reflectendo, cognoscit intellectus se et suam operationem et modum operandi et cetera quae sibi insunt».

54 Se sitúa la redacción de las cuestiones quodlibetales entre la Cuaresma de 1305 y la Cuaresma de 1307. Cf. Noone, T. B. y Roberts, H. F. “John Duns Scotus' Quodlibet” en Shabel, C. (ed.), Theological Quodlibeta in the Middle Ages: The Fourteenth Century, Brill, Leiden-Boston 2007, p. 132.

55 Duns Scotus, Quodlibet III, n. [2] 8 (ed. Alluntis, p. 93): «Ens ergo vel res isto primo accipitur omnino communissime, et extendit se ad quodcumque quod non includit contradictionem, sive sit ens rationis, hoc est praecise habens esse in intellectu considerante, sive sit ens reale, habens aliquam entitatem extra considerationem intellectus». Las cursivas son mías. 
Este texto no solamente funda un espacio intencional común al ente de razón y al ente real (o, en otros términos, a la lógica y a la metafísica); sino que también afirma, que así entendido, communissime, el ente es el objeto primero del intelecto ${ }^{56}$. En cambio, aquí, en la cuestión 5 del comentario a la Isagoge, el objeto primero del intelecto se presenta como el 'quod quid est', que entendemos como la esencia de la cosa, diferenciada de su modo universal de aprehensión.

Ya en el preámbulo citado al comienzo de este trabajo, Escoto identificó la primera acepción del término universal con el objeto propio del intelecto, allí donde el universal es tomado como sujeto, es decir, como «una cosa de primera intención a la que se aplica la intención de universal» ${ }^{57}$. En ese sentido, resulta evidente que, en este primer periodo de su pensamiento, el objeto primero del intelecto es la esencia o cosa de primera intención y no el ente entendido como «todo lo que no incluye contradicción». En una nota interpolada a este mismo pasaje, Escoto insiste una vez más en que el objeto primero del intelecto es el quod quid est de toda cosa, su naturaleza, pero aquí esta afirmación se inscribe en otra de mayor alcance, este objeto primero es aquel sobre el que tratan todas las ciencias, en tanto ellas tratan de los universales; sin embargo, la ciencia que se ocupa en grado sumo de este objeto es la metafísica ${ }^{58}$. Como conclusión preliminar, al menos puede afirmarse que en este periodo correspondiente a la redacción del comentario, Escoto aún no ha sostenido la tesis según la cual el objeto primero del intelecto es el ente communissime sumpto, como lo hará explícitamente en el Quodlibet III.

En efecto, tanto en la Ordinatio, como en el Quodlibet III, el grado de generalización que alcanza el objeto primero del intelecto se va incrementando. En la Ordinatio, el objeto primero del intelecto es el ente en tanto ente ${ }^{59}$, entendiendo por tal, un concepto unívoco capaz de cubrir tanto a Dios como a la creatura, es decir, la totalidad de lo real. Esta tesis también se inspira indirectamente, via Enrique de Gante $^{60}$, en Avicena ${ }^{61}$. Se trata de la famosísima tesis de la univocidad del ser ${ }^{62}$. Así

56 Duns Scotus, Quodlibet III, n. [2] 9 (ed. Alluntis, p. 94): «Et isto intellectu communissimo, prout res vel ens dicitur quodlibet conceptibile quod non includit contradictionem (sive illa communitas sit analogiae sive univocationis, de que non curo modo) posset poni ens primum obiectum intellectus». Las cursivas son mías.

57 Duns Scotus, In Porph., q. 4, Prooemium, (OPh, I, 21): «Ideo notandum est quod 'universale', sicut et cetera concreta, sumitur tripliciter. $<1>$ Quandoque pro subiecto, scilicet pro re primae intentionis cui applicatur intentio universalis, et hoc modo universale est primum obiectum intellectus». Las cursivas son mías.

58 Duns Scotus, In Porph., q. 4, Prooemium, (OPh, I, 21), adnotatio interpolata: «Et omnis scientia est universalium, maxime tamen metaphysica quia considerat 'quod quid est' cuiuslibet rei quod est primum obiectum intellectus».

59 Duns Scotus, Ord., I, d. 3 p. 1, qq.1-2 nn. 137-151 (Vat. III, 85-94).

60 Enrique de Gante, Summae, art. 21, q. 2 (I, 124r E): «Dictum de pluribus quod habet per se intellectum propter intellectus illorum, est aliquid reale commune ad illos, quia omnis conceptus fundatur in re aliqua. Ens est huiusmodi, quia secundum Avicennam ens "imprimitur impressione prima", etiam antequam in ipsa imprimitur intellectus aut creaturae aut Dei. Ergo, etc.».

61 Avicenna, Philosophia prima sive scientia divina, I, 5 (AvL, I, 31-33): «Dicemus igitur quod res et ens et necesse talia sunt quod statim imprimuntur in anima prima impressione, quae non acquiritur ex aliis notioribus se, [...] Quae autem promptiora ad imaginandum per seipsa, sunt ea quae communia sunt omnibus rebus, sicut res et ens et unum, et cetera. Et ideo nullo modo potest manifestari aliquod horum probatione quae non it circulari, vel per aliquid quod sit notius illis. Unde quisquis voluerit discurrere de illis incidet in involucrum, sicut ille qui dixit quod certitudo entis est quod vel est agens vel patiens: quamvis haec divisio sit entis, sed tamen ens notius est quam agens vel patiens. Omnes enim homines imaginant certitudinem entis, vel ignorant si debeat esse agens vel patiens; et mihi quousque nunc non patuit hoc nisi argumentatione tantum».

62 Sobre este punto, cf. Boulnois, O. "La destruction de l'analogie et l'instauration de la métaphysique", en Jean Duns Scot, Sur la connaissance de Dieu et l'univocité de l'étant, Presses Universitaires de France, París 1988, pp. 11- 81; Dumont, S. "Henry of Ghent and Duns Scotus" en Marenbon, J. (ed.), Routledge History of 
pues, el objeto primero del intelecto es un concepto más general que el relativo a la esencia o naturaleza.

Por su parte, en el Quodlibet III, Escoto expande a su vez este grado de generalidad al ente entendido como lo concebible sin contradicción, es decir, en un dominio que incluye por igual al ente real y al ente de razón. En el comentario a la Isagoge, en cambio, el objeto primero del intelecto es la esencia de la cosa en su desnudez, independiente de su individuación y de su universalización, pero en todo caso no en tanto concebible, sino en tanto susceptible de individualizarse o universalizarse.

\section{La naturaleza común}

Ahora bien, el comentario a la Isagoge, como lo hemos visto, restringe el tratamiento del universal y de hecho concibe el universal como objeto exclusivamente lógico. No hay universal extramental. Para un tratamiento no lógico del elemento común a la esfera extramental y a la esfera intencional, habrá que esperar la tesis escotista sobre la naturaleza común, expuesta en la Ordinatio ${ }^{63}$. Sin ánimo de exhaustividad, presentamos brevemente en lo que sigue la otra cara de la esencia, esta vez, en su capacidad de hallarse en muchos individuos, más allá de su aprehensión por el intelecto. Esta naturaleza coincide precisamente con la primera acepción del universal en el preámbulo que comentamos. De hecho, se trata de enfatizar ese elemento común de la esencia en su triple estatuto. En la Ordinatio, pues, el énfasis no cae sobre el universal como modo de concebir la esencia (modus concipiendi essentiae), sino precisamente en la esencia como naturaleza común.

Sin embargo, esta distinción sigue reposando explícitamente en la teoría aviceniana del triple estatuto de la esencia. Por ello, la introducción de la tesis escotista de la naturaleza común se construye sobre el andamiaje que separa, de un lado, la esencia, y del otro, su captación universal y su instanciación en el individuo. En un lenguaje que recuerda al de Avicena, Escoto afirma en la Ordinatio:

Ahora bien, no solo esta misma naturaleza es indiferente por sí a ser en el intelecto o en un particular, y por ello, a ser universal y particular (o singular), sino que también, ella misma, teniendo ser en el intelecto, no tiene primeramente la universalidad por sí. Pues aunque ella misma sea inteligida bajo la universalidad como bajo el modo de ser inteligida, la universalidad sin embargo no es una parte de su concepto primario, porque no es un concepto del metafísico, sino del lógico (en efecto, el lógico considera las segundas intenciones aplicadas a las primeras, según el mismo). Por ello, la primera intelección es de la naturaleza en tanto no es cointeligido ningún modo, ni lo que le pertenece en el intelecto, ni lo que le pertenece fuera del intelecto; aunque el modo de inteligir de aquel intelecto sea la universalidad, este modo no es inteligido ${ }^{64}$.

En este texto, Escoto destaca la indiferencia absoluta de la naturaleza (aquí la llama naturaleza y no esencia como en el comentario a la Isagoge). Esta indiferencia

Philosophy. Vol. III: Medieval Philosophy, Routledge, Londres 1998, pp. 131-328.

${ }^{63}$ Cf. el artículo fundamental Boulnois, O. "Réelles intentions: Nature commune et universaux selon Duns Scot", en Revue de métaphysique et de morale, 97 (1), 1992, pp. 3-33 y, del mismo autor, Lire le Principe d'individuation de Duns Scot, Vrin, París 2014, pp. 191-194.

${ }_{64}$ Duns Scotus, Ord., II, d. 3, p. 1, q.1, n. 33 (Vat. VII, 403-404). Las cursivas son mías. 
se funda sobre el contenido de «su concepto primario». En otros términos, la universalidad no está contenida dentro del concepto de la naturaleza. Por lo demás, Escoto vuelve sobre una distinción que ya hemos visto operando en el comentario a la Isagoge. El metafísico se ocupa de la naturaleza, mientras que el lógico se ocupa del universal.

Este pasaje diferencia pues la naturaleza del modo como el intelecto la concibe. El segundo paso consiste en diferenciar la naturaleza de la individualidad en la que se contrae en la realidad extramental. En otros términos, en la Ordinatio, Escoto también demuestra que la naturaleza no se identifica con sus instancias individuales. El pasaje reza así:

Y así como según aquel ser $<$ que tiene en el intelecto $>$ la naturaleza no es por sí universal, sino que la universalidad le acaece (accidit) a esta naturaleza según su primera razón, según la cual es un objeto, - así también en la realidad externa, donde la naturaleza está acompañada de la singularidad, no está aquella naturaleza determinada a la singularidad, sino que es naturalmente anterior a aquella razón que la contrae a esa misma singularidad; y en cuanto es naturalmente anterior a aquello que la contrae, no le repugna ser sin aquello que la contrae. Y así como el objeto en el intelecto según su primacía y universalidad tiene verdaderamente un ser inteligible (vere esse intelligibile), así también en la realidad la naturaleza tiene, de acuerdo a su entidad, un verdadero ser real fuera del alma (verum esse reale extra animam); y de acuerdo con esa entidad, tiene una unidad proporcional que es indiferente a la singularidad de tal modo que no le repugna a esa unidad por sí el ser puesta con cualquier unidad de singularidad (unitate singularitatis), (por lo tanto, de este modo entiendo: 'que la naturaleza tenga una unidad real, menor que la unidad numérica'); y aunque no la tenga por sí, de tal modo que esté incluida en la razón de naturaleza (porque 'la equinidad es sólo la equinidad' según Avicena en el libro V de su Metafísica $^{65}$ ), sin embargo, aquella unidad es una propiedad (passio propia) de la naturaleza según su entidad primera, y en consecuencia, ni es por sí 'esta' intrínsecamente, ni según su entidad propia necesariamente está incluida en esta misma naturaleza según su primera entidad ${ }^{66}$.

Así como a la naturaleza concebida por el intelecto le acaece la universalidad, así también a la naturaleza, en la realidad extramental, le acaece la singularidad. En ambos casos, la razón de la disociación de la naturaleza con respecto a su universalización por el intelecto y a su individuación en la realidad extramente obedece a la anterioridad definicional de la naturaleza. Y, en el caso de su individuación, a que a la naturaleza «no le repugna ser sin aquello que la contrae». En otros términos, su esencia no incluye necesariamente ese elemento contrayente que da lugar a la individuación. Sin embargo, tampoco le repugna articularse con dicho principio de singularidad. Allí, reencontramos la indiferencia de la esencia, esta vez, frente a su eventual singularización.

Ahora bien, la reflexión de Escoto lo conduce a introducir un tercer elemento hasta ahora no considerado: la comunidad. Si la universalidad es lógica y la individualidad (o singularidad) extramental, la comunidad es esencial. Es decir, la comunidad forma parte del concepto de la esencia. He aquí el pasaje.

Cf. Avicenna, Philosophia prima sive scientia divina, V, 1 (AvL, II, 228 ss.)

66 Duns Scotus, Ord., II, d. 3, p. 1, q.1, n. 34 (Vat. VII, 404-405). 
Es manifiesto que la comunidad y la singularidad no están relacionadas con la naturaleza como lo están el ser en el intelecto (esse in intellecto) y el ser verdadero fuera del alma (esse verum extra animam), puesto que la comunidad pertenece a la naturaleza fuera del intelecto, del mismo modo que pertenece la singularidad; y la comunidad pertenece a la naturaleza por sí, mientras que la singularidad le pertenece por algo en la cosa que contrae la naturaleza; pero la universalidad no pertenece a la cosa por sí. Y es por ello que concedo que debe buscarse la causa de la universalidad, pero no debe buscarse ninguna otra causa de la comunidad que la naturaleza misma. Y una vez que la comunidad es puesta en la naturaleza según su propia entidad y unidad, necesariamente debe buscarse la causa de la singularidad, la cual añade algo (superaddit aliquid) a esta naturaleza a la cual pertenece ${ }^{67}$.

Según esto, la comunidad de la naturaleza es un efecto propio de dicha naturaleza. La comunidad no es un efecto lógico del intelecto, ni un resultado adicional derivado de la contracción que da lugar a la individuación. Tanto la comunidad como la simplicidad pertenecen a la naturaleza extramental. Sin embargo, la singularidad no forma parte de la naturaleza, mientras que la comunidad sí, pues es un efecto de ella. Así vista, la comunidad de naturaleza garantiza, en el orden ontológico, la eficacia de la concepción y la predicación universal en el intelecto. Con este vínculo, Escoto evita la posible desconexión entre el mundo y la mente.

\section{Consideraciones finales}

Siguiendo la doctrina aviceniana, Duns Escoto disocia la esencia tanto de la universalidad como de la singularidad. Como hemos podido observar, este movimiento ya está presente en su comentario a la Isagoge y tendrá un rol permanente en su pensamiento; basta considerar el lugar que ocupa en el tratamiento de la tesis de la naturaleza común. La universalidad es un accidente intencional causado por el intelecto con ocasión de su intelección de la esencia. Este accidente, el universal, es el modo bajo el cual el intelecto capta la esencia, es su modus concipiendi. El universal es una intención segunda y, con ello, un objeto lógico. El comentario a la Isagoge subraya esa dimensión y la asocia al campo de estudio de la ciencia lógica. Con la perspectiva aviceniana, Escoto empleará el término «universal» para referirse únicamente a este modus concipiendi, es decir a esta operación lógica que permite la aprehensión de la esencia.

Por otro lado, la garantía de la existencia de la esencia en múltiples singulares no proviene ya de su universalidad, sino de su comunidad. Siendo la comunidad un rasgo de la esencia en tanto esencia, y no de la esencia en tanto individuada o en tanto universalizada por el intelecto. La comunidad es un rasgo intrínseco a la esencia que garantiza en el orden ontológico el acto de aprehensión y predicación universal de la esencia en el orden lógico o, mejor aún, noético. 


\section{Referencias bibliográficas}

Fuentes primarias

Aristóteles, Tratados de lógica (Órganon), II vols., introducciones, traducciones y notas de Miguel Candel Sanmartín, Gredos, Madrid 1995.

Aristóteles, Categorías/De interpretatione y Porfirio, Isagoge, introducción, traducción y notas de L. M. Valdés Villanueva, Tecnos, Madrid 2012.

Aristoteles Latinus, Categoriae vel praedicamenta. Translatio Boethii, editio composita translatio Guillelmi de Moerbeka, lemmata e Simplicii commentario decerpta, PseudoAugustini paraphrasis Themistiana, Minio-Paluello, L. (ed.), «Aristoteles Latinus, I 1-5», Desclée De Brouwer, Brujas-París 1961.

Aristoteles Latinus, Categoriarum supplementa. Porphyrius, Porphyrii Isagoge, Translatio Boethii, et Anonymi Fragmentum vulgo vocatum "Liber sex principiorum", MinioPaluello, L. y Dod, B. G. (eds.), «Aristoteles Latinus, I 6-7», Desclée De Brouwer, Brujas-París 1966.

Auctoritates Aristotelis. Un florilège médiéval: étude historique et édition critique, ed. J. Hamesse, «Philosophes Médiévaux, XVII», Publications Universitaires-Béatrice Nauwelaerts, Lovaina-París 1974.

Avicenna, Liber de philosophia prima sive Scientia divina I-IV, Van Riet, S. (ed.), introducción doctrinal de G. Verbeke, «Avicenna Latinus», Peeters-Brill, Lovaina-Leiden 1977.

Avicena, Logyca, Venetiis 1508, ff. 2-12.

Boecio, In Isagogen Commenta, S. Brandt (ed.), «Corpus Scriptorum Ecclesiasticorum Latinorum, 48», F. Tempsky y G. Freytag, Viena-Leipzig 1906.

Enrique de Gante, Summae Quaestionum Ordinariarum (Reprint of the 1520 Edition), II vols., Eligius M. Buytaert (ed.), «Franciscan Institute Publications, Texts Series 1», The Franciscan Institute-E. Nauwelaerts-F. Shöningh, Nueva York-Lovaina-Padenborn 1953.

Juan Duns Escoto, Opera omnia III: Ordinatio I, d. 3, C. Balić et al. (eds.), Typis Polyglottis, Civitas Vaticana 1954.

Juan Duns Escoto, Obras del Doctor Sutil Juan Duns Escoto: Cuestiones Cuodlibetales, edición del texto latino, introducción, resúmenes y traducción española de Félix Alluntis, Biblioteca de Autores Cristianos, Madrid 1968.

Juan Duns Escoto, Opera omnia VII: Ordinatio II, d. 1-3, C. Balić et al. (eds.), Typis Polyglottis, Civitas Vaticana 1973.

Juan Duns Escoto, Opera philosophica I: Quaestiones In Librum Porphyrii Isagoge et Quaestiones Super Praedicamenta Aristotelis, R. Andrews et al. (eds.), The Franciscan Institute, St. Bonaventure (NY) 1999.

Juan Duns Escoto, Cuestiones sobre las Categorías de Aristóteles, qq. 1-3, trad. castellana Salinas Leal, H. H. en Universitas Philosophica, 67, 2016, 275-302.

Porfirio, Isagoge, Introducción, traducción y notas de J. J. García Norro y R. Rovira, Anthropos, Barcelona 2003.

Porphyre, Isagoge, texto griego y latino, traducción francesa de A. de Libera y A.-Ph. Segonds, introducción y notas de A. de Libera, Vrin, París 1998.

Bibliografía secundaria

Boulnois, O. "La destruction de l'analogie et l'instauration de la métaphysique", en Jean Duns Scot, Sur la connaissance de Dieu et l'univocité de l'étant, Presses Universitaires de France, París 1988, pp. 11- 81. 
Boulnois, O. "Réelles intentions: Nature commune et universaux selon Duns Scot", en Revue de métaphysique et de morale, 97 (1), 1992, pp. 3-33.

Boulnois, O. "Les catégories selon Duns Scot", en M. Carbajo Núñez (ed.), Giovanni Duns Scoto: Studi e ricerche nel VII Centenario della sua morte, Vol.1, Antonianum, Roma 2008, pp. 357-377.

Boulnois, O. Lire le Principe d'individuation de Duns Scot, Vrin, París 2014.

Dahlstrom, D. O. "Signification and Logic: Scotus on Universals from a Logical Point of View", en Vivarium, 18, 1980, pp. 81-111.

Demange, D, Jean Duns Scot. La théorie du savoir, Vrin, París 2007.

Dumont, S. "Henry of Ghent and Duns Scotus" en Marenbon, J. (ed.), Routledge History of Philosophy. Vol. III: Medieval Philosophy, Routledge, Londres 1998, pp. 291-328.

Gilon, O., Indifférence de l'essence et métaphysique chez Jean Duns Scot, Éditions Ousia, Bruselas 2012.

Gilson, É. El ser y los filósofos, Ediciones Universidad de Navarra, Pamplona 2001.

Janssens, J., "Latin translations of Ibn Sīnā (Avicenna)”, en Lagerlund, H. (ed.), Encyclopaedia of Medieval Philosophy. Philosophy between 500 and 1500, Springer, Dortrecht 2011, pp. $522-527$.

Kretzmann, N., "History of Semantics", en Borchert, M. (ed.), Encyclopedia of Philosophy, 2a ed., vol. 8, Macmillan, New York 1967/2005, pp. 750-807.

Libera, A. de, La Querelle des Universaux. De Platon à la fin du Moyen Age, Seuil, París 1996.

Libera, A. de, L’art des géneralités. Théories de l'abstraction, Aubier, París 1999.

Marenbon, J., Boethius, Oxford University Press, Oxford 2003.

Noone, T. B. "Universals and Individuation", en Williams, T. (ed.), Cambridge Companion to Duns Scotus, Cambridge University Press, Cambridge 2003, pp. 100-128.

Noone, T. B. y Roberts, H. F. “John Duns Scotus' Quodlibet” en Shabel, C. (ed.), Theological Quodlibeta in the Middle Ages: The Fourteenth Century, Brill, Leiden-Boston 2007, pp. 131-198.

Newton, L1. A. "Duns Scotus's Account of a Propter Quid Science of the Categories", en Newton, L1. A. (ed.), Medieval Commentaries on Aristotle's Categories, Brill, LeidenBoston 2008, pp. 221-258.

Rijk, L. M. de, “A Study on the Medieval Intentionality Debate up to ca. 1350", en Giraldus Odonis O. F. M., Opera Philosophica Vol. II: De Intentionibus, de Rijk, L. M. (ed.), Brill, Leiden-Boston 2005, pp. 17-376.

Salinas Leal, H. H. "Observaciones sobre la constitución medieval de la lógica: de scientia sermocinalis a scientia rationalis", en Universitas Philosophica, 64 (32), enero-junio, 2015, pp. 96-111.

Salinas Leal, H. H. "La determinación de la lógica como ciencia común intencional en Duns Escoto", en Franciscanum, LVIII (65), enero-junio 2016, 51-86. http://dx.doi. org/10.21500/01201468.2183

Salinas Leal, H. H. "Naturaleza y unidad lógica de las categorías en Duns Escoto", Pensamiento: Revista de investigación e información filosófica, 73 (275), enero-abril, 2017, pp. 129-145. http://dx.doi.org/10.14422/pen.v73.i275.y2017.008 